\section{Associação entre circunferência abdominal e hipertensão arterial em mulheres: Estudo Pró-Saúde}

\author{
Association between abdominal circumference and \\ hypertension among women: the Pró-Saúde Study
}

\footnotetext{
1 Instituto de Nutrição, Universidade do Estado do Rio de Janeiro, Rio de Janeiro, Brasil. 2 Instituto de Medicina Social, Universidade do Estado do Rio de Janeiro, Rio de Janeiro, Brasil.

${ }^{3}$ Escola Nacional de Saúde Pública Sergio Arouca, Fundação Oswaldo Cruz, Rio de Janeiro, Brasil.

Correspondência M. H. Hasselmann Departamento de Nutrição Social, Instituto de Nutrição, Universidade do Estado do Rio de Janeiro. Rua São Francisco Xavier 524, 12 o andar Rio de Janeiro, $R J$ 20559-900, Brasil. hasselm@ims.uerj.br
}

\begin{abstract}
This study aimed to estimate the prevalence of hypertension according to levels of abdominal circumference (AC) and body mass index (BMI, expressed as $\mathrm{kg} / \mathrm{m}^{2}$ ) among public employees at a university in Rio de Janeiro, Brazil. Cross-sectional data were obtained for 1,743 non-pregnant women aged 24-69 years participating in the Pró-Saúde Study. Underweight women and those with $B M I \geq 35 \mathrm{~kg} / \mathrm{m}^{2}$ were excluded. Hypertension was defined as systolic blood pressure $\geq 140 \mathrm{mmHg}$ or diastolic $\geq 90 \mathrm{mmHg}$ or the use of anti-hypertensive drugs. Age-adjusted prevalence rates for hypertension were calculated considering two strata of AC (normal: $\leq 88 \mathrm{~cm}$; high: $>88 \mathrm{~cm}$ ) and three levels of BMI (normal: $18.5-24.9 \mathrm{~kg} / \mathrm{m}^{2}$; overweight: $25.0-29.9 \mathrm{~kg} / \mathrm{m}^{2}$; and obesity I: $30.0-34.9 \mathrm{~kg} / \mathrm{m}^{2}$ ). Among normal-weight women, participants with high AC showed a two-fold higher prevalence of hypertension as compared to those with $A C \leq 88 \mathrm{~cm}$ (18\% vs. 8\%, $p<0.05)$. Routine measurement of $A C$, in addition to BMI, could contribute to early identification of hypertension in women.
\end{abstract}

Abdominal Circumference; Obesity; Hypertension; Women's Health
Maria Helena Hasselmann 1

Eduardo Faerstein 2

Guilherme L. Werneck 2

Dóra Chor 3

Claudia S. Lopes 2

\section{Introdução}

Índices antropométricos distintos, em particular o índice de massa corporal (IMC) para classificação de sobrepeso e obesidade, têm sido utilizados para avaliar a associação entre excesso de peso e morbidade cardiovascular. Ainda que seja uma excelente medida de obesidade, o IMC não considera a variação na distribuição da gordura corporal 1 .

Vários estudos têm registrado que a circunferência abdominal (CA) ou da cintura (CC), indicadores de distribuição da gordura corporal, estão associadas à ocorrência de doenças metabólicas e cardiovasculares, dentre estas a hipertensão arterial 1,2,3,4,5,6,7,8. Não obstante, a literatura é controversa, havendo estudos que apontam a CA e a CC, e não o IMC, como os principais fatores associados ao risco de desfechos adversos em saúde relacionados à obesidade 2,6. Por outro lado, Zhu et al. 5 endossam a utilização da combinação de CC e IMC na identificação de fatores de risco para doenças cardiovasculares.

Buscando contribuir para esse debate, este estudo objetiva estimar a prevalência de hipertensão arterial segundo estratos de CA e IMC em uma população de funcionárias públicas de uma universidade localizada no Estado do Rio de Janeiro, Brasil. 


\section{Material e métodos}

Trata-se de estudo seccional entre 1.743 mulheres de 24 a 69 anos, funcionárias técnico-administrativas efetivas de uma universidade, participantes da fase 2 (2001) do Estudo Pró-Saúde, investigação longitudinal sobre determinantes sociais da saúde ${ }^{9}$. Foram excluídas gestantes, aposentadas, licenciadas por motivos que não os de saúde, e aquelas cedidas a outras instituições.

Foram aferidas massa corporal, estatura, CA, pressão arterial e escolaridade (categorias descritas na Tabela 1). As mensurações antropométricas e de pressão arterial foram realizadas com base em protocolos internacionais e resultados de estudos sobre a qualidade do processo de aferição foram considerados satisfatórios 10 .

A aferição de CA foi feita na altura da cicatriz umbilical 11. Devido à inexistência de pontos de corte universalmente aceitos para CA, optou-se por empregar aquele utilizado em investigação acerca da associação entre CC e risco para saúde (normal: $\leq 88 \mathrm{~cm}$; elevado: $>88 \mathrm{~cm}$ ) ${ }^{6}$.

Foram considerados três grupos com base no índice de massa corporal [IMC $=$ peso $(\mathrm{kg}) /$ estatura $\left.(\mathrm{m})^{2}\right]$ : eutrofia $\left(\mathrm{IMC}=18,5-24,9 \mathrm{~kg} / \mathrm{m}^{2}\right)$; sobrepeso (IMC $=25,0-29,9 \mathrm{~kg} / \mathrm{m}^{2}$ ); e obesidade I (IMC $=30,0-34,9 \mathrm{~kg} / \mathrm{m}^{2}$ ). Mulheres com baixo peso (IMC $<18,5 \mathrm{~kg} / \mathrm{m}^{2} ; \mathrm{N}=32$ ) ou obesidade II e III (IMC $\geq 35,0 \mathrm{~kg} / \mathrm{m}^{2} ; \mathrm{N}=130$ ) foram excluídas, visto que a totalidade delas apresentava, respectivamente, valores normais e elevados de CA.

Mulheres que apresentavam pressão sangüínea sistólica $\geq 140 \mathrm{mmHg}$ ou diastólica $\geq$

\section{Tabela 1}

Prevalência (intervalo de confiança de 95\% - IC95\%) de hipertensão arterial, gordura centralizada, sobrepeso e obesidade I, e distribuição dos níveis de escolaridade entre funcionárias de uma universidade no Rio de Janeiro, Brasil ( $N=1.743)$. Estudo Pró-Saúde, 2001.

\begin{tabular}{lcc}
\hline & Prevalência (\%) & IC95\% \\
\hline Hipertensão & 22,1 & $20,2-24,1$ \\
Circunferência abdominal > 88cm & 39,8 & $37,5-42,1$ \\
Sobrepeso & 34,3 & $32,1-36,6$ \\
Obesidade I & 13,3 & $11,7-15,0$ \\
Escolaridade & & - \\
Até 1o grau incompleto & 5,1 & - \\
1o grau completo & 11,6 & - \\
2o grau completo & 32,5 & - \\
Universitário completo & 50,9 & \\
\hline
\end{tabular}

90mmHg ou faziam uso de medicação anti-hipertensiva foram consideradas hipertensas. As prevalências de hipertensão arterial ajustadas para a idade e seus respectivos intervalos de 95\% de confiança (IC95\%) foram calculadas para os grupos de CA e IMC acima especificados, utilizando-se regressão logística. Os valores de prevalência ajustados aqui apresentados são aqueles preditos para a média da idade da população do estudo ( 40 anos). As análises foram realizadas usando-se o aplicativo Stata 9.0 (Stata Corp., College Station, Estados Unidos).

O estudo foi aprovado pelo Comitê de Ética da instituição onde a investigação foi desenvolvida.

\section{Resultados}

A Tabela 1 apresenta as prevalências de hipertensão arterial, sobrepeso e obesidade, e de CA $>88 \mathrm{~cm}$, assim como a distribuição dos níveis de escolaridade na população estudada. A prevalência de hipertensão arterial foi de $22,1 \%$, sendo que 237 (34,2\%) das participantes hipertensas apresentavam valores elevados de CA (resultado não apresentado em tabela).

Mulheres com valores elevados de CA apresentaram prevalência de hipertensão arterial 2,5 vezes mais alta do que as de CA normal (razão de prevalências - RP = 2,45; IC95\%: 2,042,94). A associação entre obesidade e hipertensão seguiu o mesmo padrão (RP = 2,32; IC95\%: 1,91-2,81) (resultados não apresentados em tabela).

A Tabela 2 apresenta a prevalência de hipertensão arterial e as médias de idade, CA e IMC segundo categorias combinadas de CA e IMC. A ocorrência de hipertensão arterial foi mais alta nos grupos com valores elevados de CA, sendo que estas diferenças foram mais marcantes e, estatisticamente significativas, nos grupos de mulheres eutróficas e com sobrepeso; ressaltase, entretanto, o número reduzido $(\mathrm{n}=4)$ de mulheres obesas com CA normal.

$\mathrm{Na}$ Tabela 3, as prevalências de hipertensão arterial são apresentadas nos estratos combinados de CA e IMC após ajuste para idade. Tanto para as mulheres eutróficas quanto para as obesas, a prevalência de hipertensão arterial foi cerca do dobro naquelas com valores elevados de CA, quando comparadas com as de CA normal, sendo que esta diferença foi estatisticamente significativa apenas para o primeiro grupo. Nas mulheres com sobrepeso, as prevalências de hipertensão foram semelhantes nos dois estratos de CA. 
Prevalência (intervalo de confiança de 95\% - IC95\%) de hipertensão arterial e médias \pm desvio-padrão de idade, circunferência abdominal (CA) e índice de massa corporal (IMC) segundo categorias de IMC e CA entre funcionárias de uma universidade no Rio de Janeiro, Brasil (N = 1.743). Estudo Pró-Saúde, 2001.

\begin{tabular}{|c|c|c|c|c|c|c|}
\hline & \multicolumn{6}{|c|}{ Categorias de IMC } \\
\hline & \multicolumn{2}{|c|}{ Eutrofia } & \multicolumn{2}{|c|}{ Sobrepeso } & \multicolumn{2}{|c|}{ Obesidade I } \\
\hline & $\begin{array}{l}\text { CA normal } \\
(n=833)\end{array}$ & $\begin{array}{l}\text { CA alta } \\
(n=80)\end{array}$ & $\begin{array}{c}\text { CA normal } \\
(n=212)\end{array}$ & $\begin{array}{l}\text { CA alta } \\
(n=387)\end{array}$ & $\begin{array}{c}\text { CA normal } \\
(n=4)\end{array}$ & $\begin{array}{l}\text { CA alta } \\
(n=227)\end{array}$ \\
\hline Hipertensão arterial & $11,7(9,6-14,1)$ * & 31,2 ** $(21,3-42,6)$ & $22,2(16,8-28,4)$ & 30,3 ** $(25,8-35,2)$ & $25,00(0,63-80,60)$ & $41,8(35,3-48,5)$ \\
\hline Idade (anos) & 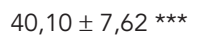 & $44,00 \pm 8,53$ & $41,20 \pm 7,32$ & $44,8 \pm 8,67$ & $44,60 \pm 7,81$ & $45,60 \pm 7,81$ \\
\hline $\mathrm{CA}(\mathrm{cm})$ & 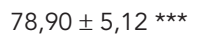 & $91,10 \pm 3,07$ & $84,30 \pm 2,82$ & $93,9 \pm 4,40$ & $84,20 \pm 2,17$ & $101,10 \pm 6,18$ \\
\hline IMC (kg/m²) & $22,3 \pm 1,6 * \star \star$ & $24,00 \pm 0,88$ & $26,30 \pm 1,03$ & $27,6 \pm 1,34$ & $31,40 \pm 1,35$ & $32,10 \pm 1,36$ \\
\hline
\end{tabular}

* IC95\% para a prevalência de hipertensão arterial;

** $\mathrm{p}$ valor < 0,05 para a comparação entre categorias de CA dentro de cada categoria específica de IMC usando o teste do qui-quadrado;

*** Média \pm desvio-padrão.

Prevalência (intervalo de confiança de 95\% - IC95\%) de hipertensão arterial ajustada para idade, segundo categorias de índice de massa corporal (IMC) e circunferência abdominal (CA) entre funcionárias de uma universidade no Rio de Janeiro, Brasil (N=1.743). Estudo Pró-Saúde, 2001.

\begin{tabular}{|c|c|c|c|c|c|c|c|c|c|c|c|c|}
\hline & \multicolumn{12}{|c|}{ Categorias de IMC } \\
\hline & \multicolumn{4}{|c|}{ Eutrofia } & \multicolumn{4}{|c|}{ Sobrepeso } & \multicolumn{4}{|c|}{ Obesidade I } \\
\hline & \multicolumn{2}{|c|}{ CA normal } & \multicolumn{2}{|c|}{ CA alta } & \multicolumn{2}{|c|}{ CA normal } & \multicolumn{2}{|c|}{ CA alta } & \multicolumn{2}{|c|}{ CA normal } & \multicolumn{2}{|c|}{ CA alta } \\
\hline & $\%$ & IC95\% & $\%$ & IC95\% & $\%$ & IC95\% & $\%$ & IC95\% & $\%$ & IC95\% & $\%$ & IC95\% \\
\hline Hipertensão arterial & 8,2 & $6,4-10,5$ * & $17,9 * \star$ & $11,1-27,6$ & 17,1 & $11,1-27,6$ & 17,5 & $13,6-22,5$ & 13,5 & $0,1-64,8$ & 25,1 & $18,4-33,5$ \\
\hline
\end{tabular}

* Intervalo de confiança para a prevalência de hipertensão arterial predita para a população feminina do estudo com idade de 40 anos;

** $\mathrm{p}$ valor < 0,05 para a comparação entre categorias de CA dentro de cada categoria específica de IMC usando o teste do qui-quadrado.

\section{Discussão}

Ainda que seja pertinente a discussão acerca do uso da CA, CC, IMC, ou da conjugação destes índices como marcadores do risco cardiovascular, observou-se na maior parte das investigações que valores elevados de CA e de CC associam-se a um risco mais elevado de hipertensão arterial $4,5,6,7,12$. Resultados semelhantes são apresentados neste artigo. De modo especial, observamos que mulheres com IMC normal (eutróficas) com CA $>88 \mathrm{~cm}$ apresentaram o dobro da prevalência de hipertensão arterial quando ajustada por idade, do que aquelas com valores abaixo deste ponto de corte.

Em concordância com nossos resultados, Janssen et al. 3 observaram prevalência de hipertensão arterial de 42,9\% entre mulheres eutróficas com valores elevados de CA, em contraste com $11,6 \%$ entre eutróficas com valores abaixo de $88 \mathrm{~cm}$. Vikram et al. 13 mostraram que a chance de ocorrência de hipertensão era quase quatro vezes maior entre mulheres não obesas (IMC < $25,0 \mathrm{~kg} / \mathrm{m}^{2}$ ) com CA $>80 \mathrm{~cm}$. Uma possível explicação para esses resultados pode estar relacionada ao papel da distribuição da adiposidade corporal. Revisão recente acerca da associação entre gordura visceral e síndrome metabólica sugere que a adiposidade abdominal é um elemento central, influenciando a resistência insulínica e, conseqüentemente, a síndrome metabólica e o risco cardiovascular 14 .

Dois comentários de cunho metodológico merecem destaque. Primeiro, em se tratando de dados transversais não é possível afirmar que valores elevados de CA aumentam o risco de desenvolver hipertensão arterial. O segundo aspecto diz respeito à inexistência de pontos de corte recomendados para discriminar CA normal e alta. Com a finalidade de buscar aplicação prática para monitoramento e identificação de grupos de risco em serviços de saúde, optou-se por utilizar 
o ponto de corte utilizado por Janssen et al. 6 para a CC, devido à sua maior especificidade como preditor de morbidade cardiovascular. De qualquer forma, análise exploratória conduzida por meio de árvores de classificação 15 identificou $\geq$ $90 \mathrm{~cm}$ como o melhor ponto de corte de CA em nossos dados para discriminar participantes hipertensas e normotensas. Ainda que o resultado dessa exploração seja aplicável somente à população estudada, é possível supor que, em função da forte correlação entre CC e CA, os valores dos pontos de corte ideais para os dois parâmetros sejam próximos.

Os resultados aqui apresentados apontam para a importância da inclusão da mensuração da CA na rotina dos serviços de saúde. A aferi-

\section{Resumo}

O objetivo deste estudo foi estimar a prevalência de hipertensão arterial segundo estratos de circunferência abdominal (CA) e índice de massa corporal (IMC, em $\mathrm{kg} / \mathrm{m}^{2}$ ) em uma população de funcionárias públicas de universidade localizada no Rio de Janeiro, Brasil. Foram analisados dados seccionais de 1.743 mulheres não grávidas de 24 a 69 anos participantes, em 2001, do Estudo Pró-Saúde. Mulheres com baixo peso ou apresentando IMC $\geq 35 \mathrm{~kg} / \mathrm{m}^{2}$ foram excluídas. $\mathrm{Mu}$ lheres que apresentavam pressão sangüinea sistólica $\geq 140 \mathrm{mmHg}$ ou diastólica $\geq 90 \mathrm{mmHg}$ ou faziam uso de medicação anti-hipertensiva foram consideradas hipertensas. A análise foi conduzida segundo dois estratos de CA (normal: $\leq 88 \mathrm{~cm}$; elevado: $>88 \mathrm{~cm}$ ) e três de IMC (eutrofia: 18,5-24,9kg/m²; sobrepeso: 25,0 $29,9 \mathrm{~kg} / \mathrm{m}^{2}$; e obesidade I: $30,0-34,9 \mathrm{~kg} / \mathrm{m}^{2}$ ). Entre $\mathrm{mu}$ lheres eutróficas, participantes com valores elevados de CA apresentaram o dobro da prevalência de hipertensão arterial do que aquelas com $C A \leq 88 \mathrm{~cm}$ (18\% vs. 8\%; $p<0,05$ ). Adicionalmente à mensuração do IMC, a aferição da CA na rotina dos serviços de saúde pode contribuir para a identificação precoce ou suspeição de hipertensão arterial.

Circunferência Abdominal; Obesidade; Hipertensão; Saúde da Mulher ção de CA, independente das medidas tradicionalmente já realizadas nesses serviços (massa corporal e estatura), poderia contribuir para a identificação precoce ou suspeição de hipertensão arterial.

A prevenção do sobrepeso e da obesidade é o principal meio para diminuir a adiposidade abdominal, ou seja, intervenções que reduzam a adiposidade total irão diminuir a CC e a CA e terão impacto nas incidências de hipertensão arterial 16. Nessa perspectiva, promover estratégias que visem a mudanças no estilo de vida, tais como aumento da atividade física, abandono do tabagismo e modificações nos hábitos alimentares - alimentação saudável - são oportunas e necessárias 17 .

\section{Colaboradores}

M. H. Hasselmann participou da idealização do artigo, da análise e interpretação dos dados e redigiu o artigo. E. Faerstein colaborou na idealização do artigo, coordenação da coleta de dados, análise e interpretação dos dados e redação final do artigo. G. L. Werneck contribuiu na coordenação da coleta de dados, análise e interpretação de dados e redigiu o artigo. D. Chor e C. S. Lopes participaram da coordenação da coleta de dados, da análise e da redação final do artigo. 


\section{Referências}

1. Lean MEJ, Han TS, Morrison CE. Waist circumference as a measure for indicating need for weight management. BMJ 1995; 311:158-61.

2. Harris MM, Stevens J, Thomas N, Schreiner P, Folsom AR. Associations of fat distribution and obesity with hypertension in a bi-ethnic population: the ARIC Study. Obes Res 2000; 8:516-24.

3. Janssen I, Katzmarzyk PT, Ross R. Body mass index, waist circumference, and health risk. Arch Intern Med 2002; 162:2074-9.

4. Velásquez-Meléndez G, Kac G, Valente JG, Tavares R, Silva CQ, Garcia ES. Evaluation of waist circumference to predict general obesity and arterial hypertension in women in Greater Metropolitan Belo Horizonte, Brasil. Cad Saúde Pública 2002; 18:765-71.

5. Zhu S, Heshka S, Wang Z, Shen W, Allison DB, Ross $\mathrm{R}$, et al. Combination of BMI and waist circumference for identifying cardiovascular risk factors in whites. Obes Res 2004; 12:633-45.

6. Janssen I, Katzmarzyk PT, Ross R. Waist circumference and not body mass index explains obesity-related health risk. Am J Clin Nutr 2004; 79:379-84.

7. Olinto MT, Nacul LC, Gigante DP, Costa JS, Menezes AM, Macedo S. Waist circumference as a determinant of hypertension and diabetes in Brazilian women: a population-based study. Public Health Nutr 2004; 7:629-35.

8. Ross R, Berentzen T, Bradshaw AJ, Janssen I, Kahn HS, Katzmarzyk PT, et al. Does the relationship between waist circumference, morbidity and mortality depend on measurement protocol for waist circumference? Obes Rev 2007; [Epub ahead of print].
9. Faerstein E, Chor D, Lopes CS, Werneck GL. Estudo Pró-Saúde: características gerais e aspectos metodológicos. Rev Bras Epidemiol 2005; 8:454-66.

10. Faerstein E, Chor D, Griep RH, Alves MG, Werneck GL, Lopes CS. Aferição da pressão arterial: experiência de treinamento de pessoal e controle de qualidade no Estudo Pró-Saúde. Cad Saúde Pública 2006; 22:1997-2002.

11. Lohman TG, Roche AF, Martorell R. Anthropometric standardization reference manual. Champaign: Human Kinetics Book; 1998.

12. Rezende FAC, Rosado LEFLP, Ribeiro RCL, Vidigal FC, Vasques ACJ, Bonard IS, et al. Índice de massa corporal e circunferência abdominal: associação com fatores de risco cardiovascular. Arq Bras Cardiol 2006; 87:728-34.

13. Vikram NK, Pandey RM, Misra A, Sharma R, Devi JR, Khanna N. Non-obese (body mass index < $25 \mathrm{~kg} / \mathrm{m} 2$ ) Asian Indians with normal waist circumference have high cardiovascular risk. Nutrition 2003; 19:503-9.

14. Ribeiro Filho FF, Mariosa LS, Ferreira SR, Zanella MT. Gordura visceral e síndrome metabólica: mais que uma simples associação. Arq Bras Endocrinol Metab 2006; 50:230-8.

15. Clark LA, Pregibon D. Tree-based models. In: Chambers JM, Hastie TJ, editors. Statistical models in S. New York: Chapman \& Hall; 1993. p. 377-419.

16. Hajjar I, Kotchen JM, Kotchen TA. Hypertension: trends in prevalence, incidence, and control. Annu Rev Public Health 2006; 27:465-90.

17. World Health Organization. Reducing risks, promoting healthy life. Geneva: World Health Organization; 2002.

Recebido em 30/Jul/2007

Versão final reapresentada em 11/Jan/2008

Aprovado em 22/Jan/2008 